\title{
SEEDS : Strategic Explorations of Exoplanets and Disks with Subaru
}

\author{
Motohide Tamura ${ }^{1}$ and the SEEDS team ${ }^{2}$ \\ ${ }^{1}$ Dept. of Astronomy, Graduate School of Science, The University of Tokyo, \\ 7-3-1 Hongo, Bunkyo-ku, Tokyo 113-0033, Japan \\ email: motohide.tamura@nao.ac.jp / motohide.tamura@astron.s.u-tokyo.ac.jp
}

\begin{abstract}
SEEDS is the first Subaru Strategic Program, whose aim is to conduct a direct imaging survey for giant planets as well as protoplanetary/debris disks at a few to a few tens of AU region around 500 nearby solar-type or more massive young stars devoting 120 Subaru nights for 5 years. The targets are composed of five categories spanning the ages of $\sim 1 \mathrm{Myr}$ to $\sim 1$ Gyr. Some RV-planet targets with older ages are also observed. The survey employs the new high-contrast instrument HiCIAO, a successor of the previous NIR coronagraph camera CIAO for the Subaru Telescope. We describe the outline of this survey and present its first three years of results. The survey has published $\sim 20$ refereed papers by now. The main results are as follows: (1) detection and characterization of the most unequivocal and lowest-mass planet via direct imaging. (2) detection of a super-Jupiter around the most massive star ever imaged, (3) detection of companions around a retrograde exoplanet system, which supports the Kozai mechanism for the origin of retrograde orbit (not in this proceedings, but see Narita et al. 2010, 2012). We also report (4) the discovery of unprecedentedly detailed structures of more than a dozen of protoplanetary disks and some debris disks. The detected structures such as wide gaps and spirals arms of a Solar-system scale could be signpost of planet.
\end{abstract}

Keywords. exoplanet; circumstellar disks; coronagraph, infrared

\section{Introduction}

The first 18 years of indirect observations have provided us more 850 exoplanets and more than 2500 promising candidates from the Kepler mission. This success has largely been due to the accurate radial velocity (RV) method and the transit (TR) method. However, these discoveries are inherently limited in several ways: Since both RV and TR studies are confined to the inner regions of exoplanetary systems $(<6$ au for a 15-yr survey), we still know very little about the planetary constituents in the outer regions. Both of the above indirect surveys are complicated due to the high level of intrinsic stellar activity for stars; thus, they have traditionally targeted old and quiet stars. Furthermore, they are also not suitable for planet searches around massive stars due to the paucity of stellar absorption lines and the reduced amplitude of the RV motion or the reduced TR depth.

In contrast to the RV or TR technique, direct imaging can be applied to both young and old stars, and can allow measurements of colors, luminosities and spectra, thereby providing temperatures and compositions. As a result, direct imaging is currently the best way to investigate Jupiter-mass or massive planets in the outer $(>5 \mathrm{au})$ regions around "young" stars.

Furthermore, recent 8-meter-class telescopes with adaptive optics (AO) and HST have had revealed the complexity and diversity of protoplanetary disks around very young stars at a scale greater than 100 au (e.g., spirals, banana-split). However, its inner regions $(\mathrm{r}<100 \mathrm{au})$ have not been explored due to a limited contrast. 
With this background, the Subaru Telescope is promoting the directly imaging project of exoplanets and their formation sites since 2009 for 5 years. This project is named SEEDS (Strategic Explorations of Exoplanets and Disks with Subaru Telescope) and is the first Subaru Strategic Program, a new open use category. With using both new coronagraph camera HiCIAO (Tamura et al. 2006; Hodapp et al. 2006; Suzuki et al. in prep.) commissioned in 2009 and adaptive optics AO188, it will observe exoplanets, companions, and circumstellar structures around about 500 stars in total. HiCIAO utilizes one $2 \mathrm{k} \times 2 \mathrm{k} \mathrm{HgCdTe}$ array with a pixel scale of $\sim 0.01$ arcsec/pixel. The ASIC "Sidecar" array controller allows us very flexible readout. HiCIAO contrast is one order better than CIAO.

SEEDS is a Japanese-led but international project; it consists of some 120 members; $2 / 3$ are domestic researchers, and $1 / 3$ are in US and EU. The project is managed by The University of Tokyo and NAOJ. The completeness and uniformity of this systematic survey will provide important statistical results to be obtained, as well as enabling the study of individual objects of particular interest. The SEEDS data set will be a dominant one in this important field of research for a period of many years.

\section{SEEDS Targets}

We have selected three main kinds of targets for planet searches (YSOs, open cluster stars, and nearby main-sequence stars) and two for disk/planet searches (protoplanetary and debris disks). About 100 targets are selected for each category. The three categories for planet searches are intended to cover the age ranges of 1-10 Myr, $100 \mathrm{Myr}$ (see Yamamoto et al. 2013), and 100-1000 Myr, respectively. See Janson et al. (2013), Thalmann et al. (2011, 2013) for debris disk targets and Brandt et al. (2013b) for moving group targets. See Narita et al. (2010, 2012) for RV targets. SEEDS observations can be conducted with the full use of new observing modes for the Subaru telescope: polarization differential imaging (PDI), spectral differential imaging (SDI) modes, and angular differential imaging (ADI) mode. The basic concept of such differential imaging (PDI/SDI) is to split up the image into two or more images and then use either different planes of polarization or different spectral filter band-passes to produce a signal that distinguishes faint objects near a bright central object from scattered halo or residual speckles. Great care is being taken to minimize the non-common-path error of the differential optics and its critical components (single and double Wollaston prisms made from high-quality birefringent YLF). However, most observations utilize ADI modes or PDI+ADI mode for a survey because of the sensitivity limit in the SDI mode. See also Brandt et al. (2013) for the data reduction.

\section{Planet results}

SEEDS has reported three (candidate) planets so far. The first one is GJ $758 \mathrm{~b}$ (Thalmann et al. 2009; Janson et al. 2011) and the second one is kappa And b (Carson et al. 2013). The former is $10-30 \mathrm{M}_{\mathrm{J}}$ companion orbiting around a Sun-like star and the latter is $\sim 13 \mathrm{M}_{\mathrm{J}}$ planet around a B star. The latter is the planet imaged around the most massive star. The third one, which is mainly described here, is GJ $504 \mathrm{~b}$ (Kuzuhara et al. 2013). This is a very faint planet orbiting a Sun-like star. It is at a distance about 18 pc from Earth. The central star itself is bright, visible to naked-eye ( $\sim 5$ mag). However, the planet is very dim, 17-20 mag at infrared wavelengths (JHKL'). The age of the central star is estimated from the gyrochronology to be 100-300 Myr, and 100-500 Myr (including other methods) even in the most conservative case. It is most likely that the 
planet mass is only 3-5.5 Jupiter masses, estimated from its luminosity and age. If so, it can be the lightest-mass planet ever imaged.

From seven epochs of observations, GJ 504b has been confirmed not to be a background stars but in fact orbits around GJ 504A. The apparent distance between the central star and planet is $44 \mathrm{au}$. Subsequent JHKL' observations have also reveal that the planet has a unique color. This provides useful information of its atmosphere. It appears that GJ 504b has less cloudy atmosphere, while other imaged planets have cloudy atmosphere.

In direct imaging, planet masses are estimated from its luminosity and age. All the imaged planets so far are younger than 50 million years, which introduce uncertainties in mass estimates. This is because we do not know the planet origin and resultantly the models we use could provide different mass estimates. Therefore, if we simply use the $\sim 14$ Jupiter-mass mass boundary between planets and brown dwarfs, their distinction suffers from this uncertainty. For example, if we consider all the imaged planets are formed in a low-energy (more accurately entropy) state, they are all more than 14 Jupiter masses. On the other hand the planet age is old enough, this uncertainty is mitigated. Since the age of GJ 504 is old enough (100-500 Myr), either model predicts a planetary mass rather than a brown dwarf mass. If we simply use the conventional hot-start model and the age estimate based on stellar rotation, the planet mass of GJ 504b is as small as 3 Jupiter masses.

How such a giant planet around Sun-like star formed? The standard planet formation model based on our Solar system is thought to be difficult to explain the formation of these outer planets. It is likely they cannot obtain enough mass in such outer regions. It is also unlikely that a new model of gravitational instability can form outer planets because they require very massive disks around Sun-like stars. Here we note that we have detected many gaps and spiral-arm structures at the same radial distance in the disks where outer planets discovered. These structures can be formed by the presence of planets within the disks. Therefore, the formation of outer planets can be possible (at least at or near its birth), one can guess. Therefore, we consider that now is a time for revisit the planet formation theory to include the results of outer planets and disk structures as SEEDS results demonstrate.

\section{Disk results}

SEEDS has detected interesting fine-structures of the disks around dozens of young stars so far; they show gaps, spiral arms, rings, and so on with a similar radial distance where those "outer" planets imaged. These structures can be considered as "signpost of planets". Our results on disks also support a requirement of a new planet formation model. Fine structures of many disks are revealed in AB Aur, LkCa 15, HD 169142, SAO 206462, PDS 70, UX Tau, and Upper Sco J1604 (protoplanetary disks), HR 4796A and HIP 79977 (debris disks). For AB Aur (Hashimoto et al. 2011), complicated and asymmetrical structures are seen in the inner part $(\mathrm{r}<140 \mathrm{au})$ of the disk while confirming the previously reported outer $(r>200 \mathrm{au})$ spiral structure. A double ring structure at $\sim 40$ and $\sim 100$ au and a ring-like gap between the two are discovered. The observed structures, including a bumpy double ring, a ring-like gap, and a warped disk in the innermost regions, provide essential information for understanding the formation mechanism of wide-orbit $(\mathrm{r}>20 \mathrm{au}$ ) planets. For LkCa 15 (Thalmann et al. 2010), sharp elliptical contours delimiting the nebulosity on the inside as well as the outside, consistent with the shape, size, ellipticity, and orientation of starlight reflected from the far-side disk wall, whereas the near-side wall is shielded from view by the disks optically thick bulk. This discovery provides confirmation of the disk geometry that has been proposed to 
explain the spectral energy distributions of such systems, comprising an optically thick disk with an inner truncation radius of 4-6 au enclosing a largely evacuated gap. An offset of the nebulosity contours along the major axis is also seen. This reinforces the leading theory that dynamical clearing by at least one orbiting body is the cause of the gap. For SAO 169142 (Muto et al. 2012), two small-scale spiral structures lying within $0.5^{\prime \prime}(70 \mathrm{au})$ are discovered for the first time. Models for the spiral structures using the spiral density wave theory are used to derive a disk aspect ratio of $\mathrm{h} \sim 0.1$. This model can potentially give estimates of the temperature and rotation profiles of the disk based on dynamical processes, independently from sub-mm observations. It also predicts the evolution of the spiral structures, which can be observable on timescales of 10-20 years, providing conclusive tests of the model. Assuming a planet, we can make predictions on the locations and, possibly, the masses of the unseen planets. For PDS 70 (Hashimoto et al. 2012), a weak-lined T Tauri star of K5 type, $0.82 \mathrm{M}_{\mathrm{SUN}}$ mass, <10 Myr age, taking into account the presence of the large and sharp gap, we suggest that the gap could be formed by dynamical interactions of sub-stellar companions or multiple unseen giant planets in the gap. A generic disk model that can simultaneously account for the general features in Spitzer, SMA, and Subaru observations. Particularly, the scattered light images for this model are computed, which agree with the general trend seen in Subaru data. Decoupling between the spatial distributions of the $\mu \mathrm{m}$-sized dust and mm-sized dust inside the cavity is suggested by the model (Dong et al. 2012a,b). See also Takami et al. (2013), Follette et al. (2013), Grady et al. (2013), Tanii et al. (2012), Mayama et al. (2012), and Kusakabe et al. (2012).

\section{References}

Brandt, T. D. et al. 2013a, ApJ, 764, 183

Brandt, T. D. et al. 2013b, ApJ in press

Carson, J. et al. 2013, ApJ, 763, L32

Dong, R. et al. 2012a, ApJ, 750, 161

Dong, R. et al. 2012b, ApJ, 760, 111

Follette, K. et al. 2013, ApJ, 767, 10

Grady, C. et al. 2013, ApJ, 762, 48

Hashimoto, J. et al. 2011, ApJ, 729, L17

Hashimoto, J. et al. 2012, ApJ, 758, L19

Hodapp, K. et al. 2006, Proc. SPIE, 6269, 62693V

Janson, M. et al. 2011, ApJ, 728, 85

Janson, M. et al. 2013, ApJ, in press

Kusakabe, N. et al. 2012, ApJ, 753, 153

Kuzuhara, M. et al. 2013, ApJ, in press

Mayama, S. et al. 2012, ApJ, 760, L26

Muto, T. et al. 2012, ApJ, 748, L22

Narita, N. et al. 2010, PASJ, 62, 779

Narita, N. et al. 2012, PASJ, 64, 124

Tamura, M. et al. 2006, Proc. SPIE, 6269, 62690V

Takami, M. et al. 2013, ApJ, 772, 145

Tanii, R. et al. 2012, PASJ, 64, 124

Thalmann, C. et al. 2012, ApJ, 707, L123

Thalmann, C. et al. 2010, ApJ, 718, L87

Thalmann, C. et al. 2011, ApJ, 743, L6

Thalmann, C. et al. 2013, ApJ, 763, L29

Yamamoto, K. et al. 2013, PASJ, in press 


\section{Discussion}

LIN: In addition to HAT-P-7, were distant stellar or planetary companions discovered around the host stars of any other hot Jupiters?

TAMURA: To my knowledge, HAT-P-7 b is so far the only case with a proper motion confirmation.

KavelaARs: Can you comment on the statistical likelihood of your one detection of a few $M_{J}$ planet at $40 \mathrm{AU}$ around a sun-like star?

TAmurA: Yes. This is a relatively young star, around $200 \mathrm{Myr}$, and from our survey we would say such systems are very rare. 